\title{
АНАЛИЗ ФРАКТАЛЬНОЙ СТРУКТУРЫ ТЕРМОГРАММ МОЛОЧНЫХ ЖЕЛЕЗ
}

\author{
ю. Е. Лях, В. Г. Гурьянов ${ }^{1}$, Е. А. Якобсон \\ Восточноевропейский национальный университет имени Леси Украинки \\ ${ }^{1}$ Национальный медицинский университет имени А. А. Богомольца
}

Термография молочных желез дает возможность определить опухоль на ранней стадии, но методы анализа термограмм нуждаются в совершенствовании. Наше исследование подтверждает целесообразность использования показателя Херста для оценки различий между термограммой здоровой молочной железы и молочной железы, пораженной опухолевым процессом.

\section{FRACTAL DIMENSIONALITY ANALYSIS OF MAMMARY GLAND THERMOGRAMS}

\author{
Yu. E. Lyah, V. G. Guryanov' ${ }^{1}$, E. A. Yakobson \\ Eastern National University Lesya Ukrainian \\ ${ }^{1}$ Bogomolets National Medical University
}

\begin{abstract}
Thermography may enable early detection of a cancer tumour within a mammary gland at an early, treatable stage of the illness, but thermogram analysis methods must be developed to achieve this goal. This study analyses the feasibility of applying the Hurst exponent readings algorithm for evaluation of the high dimensionality fractals to reveal any possible difference between normal thermograms (NT) and malignant thermograms (MT).
\end{abstract}

Рак молочной железы (РМЖ) является одной из основных причин смертности женского населения. В настоящее время наряду с совершенствованием методов лечения большое внимание уделяется ранней диагностике, которая улучшает показатели выживаемости больных.

В странах, где проводится национальная программа маммографического скрининга, отмечается снижение смертности на 30\%. Маммография является золотым стандартом в диагностике непальпируемых опухолей.

В настоящей работе приводится описание метода анализа фрактальной структуры термограмм, который может быть использован для диагностики рака молочных желез.

Термография молочных желез может определить наличие опухоли на самых ранних стадиях. В настоящее время доказано, что наличие аномальной термограммы является в 10 раз более существенным фактором риска возникновения в будущем раковой опухоли, чем наследственный фактор. Таким образом, термография является информативным индикатором риска развития раковой опухоли молочных желез.
Учитывая, что при развитии опухоли происходит формирование областей с различной температурой, важным фактором при оценке состояния молочной железы является оценка структуры поля температур.

Цель работы: показать возможности указанного метода при ранней диагностике рака молочной железы

Методы и материалы. Для регистрации термограмм применялся термограф ТКЦ-1 - прибор, измеряющий контактным способом абсолютное значение и градиенты температуры на большой площади поверхности тела и представляющий результаты измерений как в виде числовых массивов, так и карт распределения температуры по поверхности обследуемого участка тела.

Результаты и их обсуждение. Для изучения и анализа термограмм нами предлагается проведение оценки структуры поля температур с использованием алгоритма анализа фракталов высокой размерности.

Пусть $t\left(i_{1}, i_{2}\right)$ - значение температуры на двумерной поверхности в точке квадратной сетки с координатами $i_{1}$ и $i_{2}$. Поле температур разбивается на квадраты размером n x n (значение п может изменяться от минимального размера квадрата 
разбиения $\mathbf{n}=2$ до максимального $\mathbf{n}=\mathbf{n}_{\text {max }}$, для каждого квадрата среднее значение температуры $\overline{t\left(i_{1}, i_{2}\right)}$ рассчитывается по формуле:

$$
\overline{t_{n, n}\left(i_{1}, i_{2}\right)}=\frac{1}{n^{2}} \sum_{k_{1}=0}^{n-1} \sum_{k=0}^{n-1} t\left(i_{1}+k_{1}, i_{2}+k_{2}\right)
$$

Для каждого разбиения $\mathrm{nxn}$ поля температур рассчитывается значение обобщенной дисперсии $\sigma_{D M A}^{2}(n):$

$$
\sigma_{D M A}^{2}(n)=\frac{1}{N^{2}} \sum_{i_{I}=1}^{N} \sum_{i_{1}=1}^{N}\left[t\left(i_{1}, i_{2}\right)-\overline{t_{n}\left(i_{1}, i_{2}\right)}\right]^{2}
$$

где $\mathrm{N}$ - количество точек измерения на поверхности молочной железы.

Для фрактальной Броуновской функции $t(i 1, i 2)$ обобщенная дисперсия связана с размером разбивки nхn соотношением:

$$
\sigma_{D M A}^{2}(n) \sim[n]^{2 H}
$$

где Н - показатель Херста, характеризующий поведение распределения поля температур на двумерной поверхности.
На основании данного алгоритма разработана программа расчета показателя Херста для двухмерной поверхности.

При анализе термограмм было установлено различие поведения обобщенной дисперсии $\sigma_{D M A}^{2}(n)$ для термомаммограмм здоровых женщин и при наличии патологии.

Выводы. Фрактальный анализ позволяет выявить изменения температуры на термограммах, которые свидетельствуют о развитии патологии. Описанный подход позволит диагностировать РМЖ на ранних стадиях заболевания. 